\title{
Smart Charging of Electric Vehicles Considering User Behavior
}

\author{
Sulabh Sachan \\ EED, MJP Rohilkhand University \\ Bareilly \\ sulabh.iitr11@gmail.com
}

\author{
Lalit Kumar \\ CEMSE, KAUST, Saudi Arabia \\ 23955-6900 \\ lalitnbd@gmail.com
}

\author{
Sanchari Deb \\ VTT Technical Research Centre \\ Finland \\ sancharideb@yahoo.co.in
}

\begin{abstract}
To reduce the vehicle energy consumption and vehicle emissions, electric vehicles have been promoted with great efforts in India. Previous studies always focused on the adoption of electric vehicles and the layout planning of charging facilities in a certain area lack of long-term analysis of electric vehicle charging demand development in India. This paper proposes a real time price competitive market structure based on game theory for charging stations (CSs) considering various practical parameters including the waiting time and reputation function. A game theory based CS neighborhood competition model is used to select the charging price that would maximize the profit. Whereas, a CS selection game theory model is proposed taking into consideration proximity of the CS to the $\mathrm{EV}$, number of chargers and a variety of practical and physical parameters. Furthermore, a comprehensive sensitivity analysis is conducted to study their impacts on the proposed pricing mechanism.
\end{abstract}

Keywords-Distribution System Operator (DSO), Charging Stations (CSs), Electric Vehicles (EVs).

$\begin{array}{cl}\begin{array}{c}\text { Nomenclature } \\ \text { Notation }\end{array} & \text { Description } \\ w_{j} & \text { Waiting time at } j^{\text {th }} C S \\ R_{j}^{t} & \text { Reputation of } j^{\text {th }} C S \text { at time } t \\ o b j_{i}^{E V} & \text { Objective function of } i^{\text {th }} E V \\ o b j_{j}^{C S} & \text { Objective function of } j^{\text {th }} C S \\ \pi_{j}(.) & \text { Payoff function of } j^{\text {th }} C S\end{array}$

\section{INTRODUCTION}

Electric mobility is developing at a rapid pace, which is a common selected strategy for energy conservation and emission reduction in India. The total number of electric vehicles is more than 2.1 million in 2018, almost half of the world's electric car fleet (IEA, 2019). Previous studies mainly focused on the consumer adoption preference for electric vehicles and the charging infrastructure layout planning or optimization [1]. Aside from the economic and environmental benefits associated with the penetration of EVs into the roads, technical challenges are anticipated from the grid perspective. In a similar fashion to fuel stations required for fueling conventional vehicles, charging stations (CSs) are necessary to charge EVs. The importance of the CSs can be justified as follows:

- A cheaper/incentivized tariff for EV charging can be provided at CS as compared to the residential/domestic electricity tariff [2]. Also, the fast charging facility provision can be better equipped at CS with commercial/industrial high voltage lines as compared to the low voltage connections at residential/domestic level.
- An EV may require power in between trips/commutes and therefore requires immediate charging at CSs. The same objective is considered for the charging station placement problem [3] and local energy storage provision at CSs [45].

Extensive research in the literature attempted to describe the behavior between CSs. For example, in [6], the author proposed a stochastic game theory approach to manage high dynamic behavior of EVs at CS. The dynamic nature of EV increases the uncertainty in the availability and charging demand of EVs. To study the competitive behavior of CSs, a non-cooperative game theory based framework is proposed where each CS tries to increase the profit by optimizing charging price and selected price information is sent to the EVs to decide whether to charge or not [7]. Similarly, the interaction between EVs and CSs was studied using various game theory models such as mean field game [8], congestion game [9], super-modular game [10] and potential game [11].

From the EV perspective, the CS selection problem is handled differently according to literature. One way to handle this problem is by searching for the optimal path of the vehicle. In this type of approach, various parameters are considered such as the waiting time, the electric network congestion and the electricity prices at different CSs. In [12], the authors' goal was to reduce the EV traveling time and traffic impediments accounting for accidents through an evolutionary game theory approach. Similarly, vehicle route was optimized with an objective of minimizing the total distribution costs subject to various constraints such as battery capacity, charging time delivery/pickup demands and loading on the vehicle [13]- [17]. This paper proposes a non-cooperative game theory based price adjustment game between CSs. Aim of this work, to study and formulate a CS problem with consideration of various practical parameters. Although some of previous work has considered price competitive game between CSs to maximize the sale of electricity [18], [19] to the best of our knowledge none of the previous works considers the various parameters that affect the price game. Such as, waiting time and reputation of CS in the competitive game also effect of competition between different sizes of the CSs. However, in real scenario, all these parameters will affect the operation of CSs. Therefore, these parameters are also necessary to model and incorporate in the price game. Moreover, it is very difficult to solve this price comparative game in closed form. This is also a challenge to design a real/practical system.

In this work, we formulate the price competition game between different CSs with the consideration of the electric distribution network, real time price and analyzed the various characteristics of CSs. The main contributions of our work are as follows: 
- $\quad$ Proposed, a real time competitive price game between CSs using non-cooperative game theory approach. Proposed model also consider waiting time and reputation function. Also, considered the real time electricity price to the CSs.

- Prove the existence of Nash equilibrium along with unique Nash equilibrium also explained.

\section{PROBLEM FoRMULATION}

This section focuses on modeling all the parameters affecting the price game for EVs and CSs. The selected schematic model for price game is presented in Fig. 1. In this each CS optimize charging price in order to maximize profit. To study this process modeling of two directly related components are required described as following:

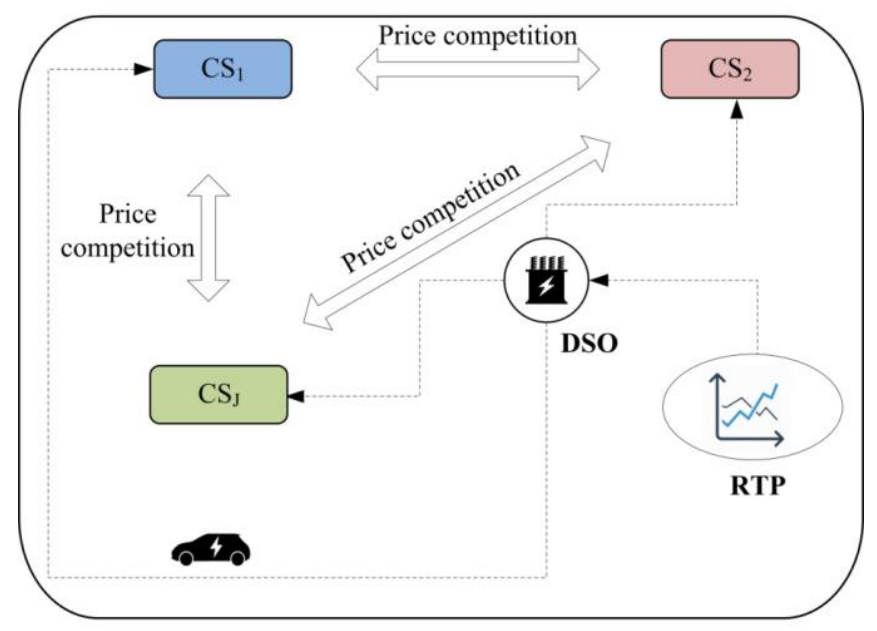

Fig. 1. System model replicates the operation of CSs.

\section{A. Electric Vehicles}

The goal of this paper is to understand the CSs behavior. Only EVs that can charge at CSs are considered for the purpose of the study. Other charging option such as charging at home is not considered in this study. EVs are assumed to be uniformly distributed on the road as in [20]. The EV's selection of CS accounts for a variety of factors such as the offered price by CSs, the distance from CSs, the expected waiting time required for charging EV and the reputation of CSs that is newly described in this work. In the realistic scenario, the distance between $j^{\text {th }} \mathrm{CS}$ and $i^{\text {th }} \mathrm{EV}\left(d_{i, j}\right)$ is a very important parameter for the selection of CS as it can increase the driving time and required charging. Therefore, each $\mathrm{EV}$ tries to reduce the distance in the objective function. Similarly, the offered charging price by the $j^{\text {th }} \mathrm{CS}\left(p_{j}\right)$ is also important in the competitive game. Each EV tries to reduce the charging cost and distance. Considerations of these two parameters can be written for $i^{\text {th }} \mathrm{EV}$ as following:

$$
o b j_{i}=\underset{j \in J}{\operatorname{argmin}}\left(\varphi_{1} \cdot p_{j}+\varphi_{2} \cdot d_{i, j}\right)
$$

The formulation of waiting time is done with the consideration of EVs and CS behavior on $t^{\text {th }}$ time interval. Mainly, there are few parameters that directly affect the waiting time such as EVs arrival at CS, EV average required power $P_{i}$, Number of charger $N_{j}^{s}$ and charging rate $C R_{j}$. We assume that $d_{i, j} \times \lambda^{t}$ EVs are approaching the $j^{t h}$ CS to charge their EVs at time $t$. The average waiting time $w_{i, j}$ will increase with increase in the required power $\left(d_{i, j} \times \lambda^{t} \times P_{i}\right)$. Whereas, number of charger $\left(N_{j}^{S}\right)$ and charging rate $\left(C R_{j}\right)$ will reduce the average waiting time. This phenomena can be formulated as the following:

$$
w_{i, j}=\frac{d_{i, j} \times \lambda^{t} \times P_{i}}{N_{j}^{S} \times C R_{j}}
$$

Note that we use $\lambda^{t}$ to denote the EV density $(\mathrm{EVs} / \mathrm{km})$ on road because it changes with respect to time.

\section{PRofit MAXIMIZATION}

With the substantial increase in the number of EVs and the acceleration of the pace of life, people will inevitably put forward higher requirements for the charging time and charging power of EVs in the future, which directly affects people's satisfaction and expectations for EVs. Under this model, CSs are owned by different owners. Therefore, each CS will try to maximize its benefit independently and selfish in price selection to maximize its own profit. Therefore, we formulated this price competition between CSs as a non-cooperative game where each CS makes the decision independently. An each CS tries to increase its profit by varying the charging price. Therefore, we formulated this price competition between CSs as a noncooperative game where each CS makes the decision independently. The total revenue by the $j^{t h} \operatorname{CS} \pi_{j}\left(p_{j}, p_{-j}\right)$ is presented as follows:

$$
\pi_{j}\left(p_{j}, p_{-j}\right)=\left(p_{P}^{t}-p_{j}^{t}-\gamma_{j}^{t}\right) \times N_{j}^{E V}(t) \times P_{j}^{t}
$$

In the paper, we assume that each CS will try to use the profit maximization algorithm to increase its profit. Therefore, each CS solves the following profit maximization problem presented in terms of the price of charging $p_{j}$.

$$
\max _{p_{j} \in \mathbb{R}^{+}} \pi_{j}\left(p_{j}, p_{-j}\right)
$$

\section{RESULTS AND DISCUSSION}

In this section, the simulation of price game is illustrated with the change in the various parameters are also investigated and the effect of new parameters is observed.

\section{A. Test System}

In this study, a realistic environment is considered to study the behavior of the charging game. The road network of Guwahati City, shown in Fig. (2), is considered in this paper for the purpose of the study. The dimension of the area under study is $7 \mathrm{~km}$ width $\times 4 \mathrm{~km}$ length. The locations of CSs are

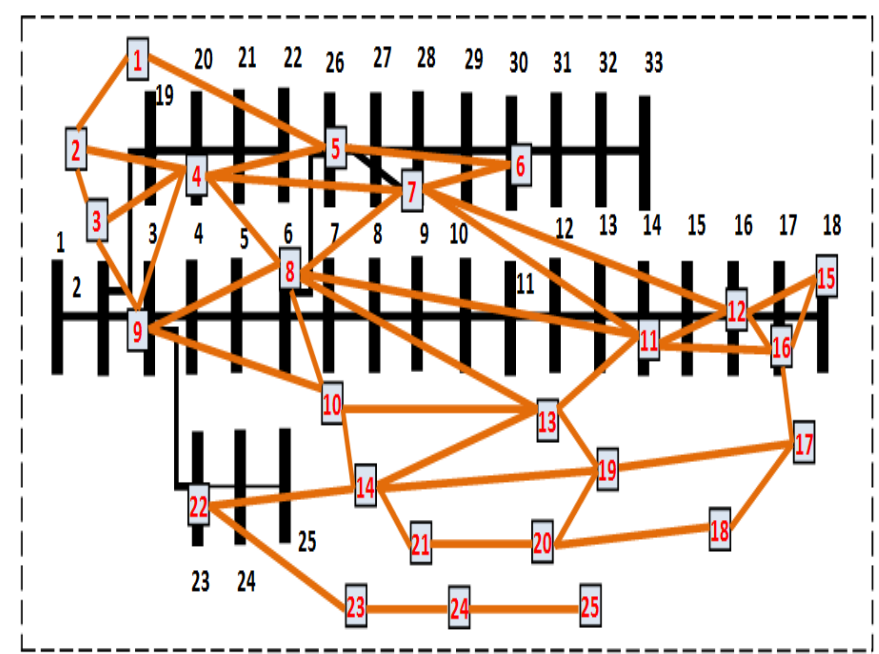

Fig. 2. Presence of CSs on road network and electrical network. 
assumed to be the place of both existing conventional fuel station and current CS. Each CS can be approach by EV via different routes that are considered part of the charging game. It is assumed that each CS would have a different capacity or charging port available $\{2,4,6,8\}$. The charging rate $(\mathrm{CR})$ is also variable and controlled by CSs owner to maximize their profit. For this study, the CR is constant for 1 day. This study also considered the average probability of the availability of EV on road [20] which is shown in Fig. (3) with standard deviation of $8 \%$.

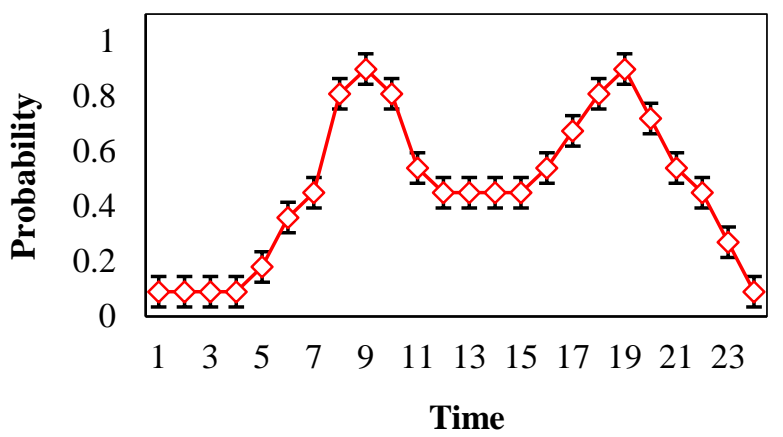

Fig. 3. Probability of availability of EV on road

\section{B. Performance Evaluation}

To study the impact of the price competition game between CSs different case studies, accounting for evenly distributed EVs on the roads, are considered. The case studies are simulated for 30 days under similar parameters and environment to study the impact of the reputation function on the proposed formulation. The simulated case studies target different objectives named as i) Without reputation function, ii) With reputation function and iii) With reputation and DSO incentive during peak hours.

For the second case study (incorporating the reputation function), the considered reputation function derived in this work and its impact on the charging game can be seen in Fig. 4. The profit made by CS 4 has reduced by approximately $11 \%$ as CS 4 has less reputation compared to CS 1 . The number of EV moving towards CS 3 and CS 4 has dropped down to $63.1 \%$ which seems to be less competed to the Case $1 \mathrm{EVs}$ movement. Whereas, CS 2 profit is improved because of its high reputation.

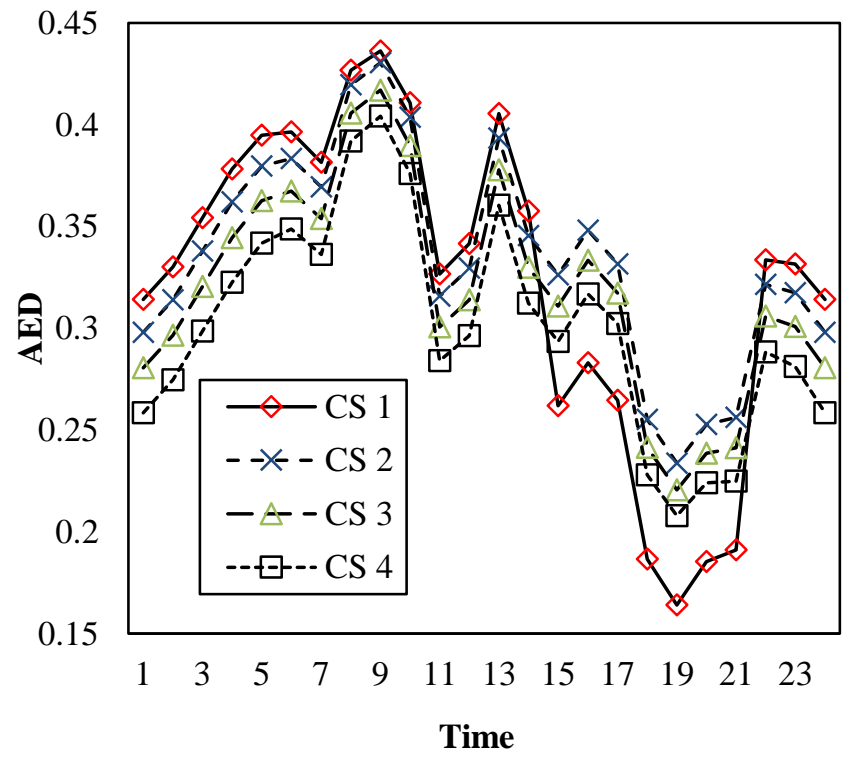

Fig. 4. A typical day selected price by CSs for Case 3

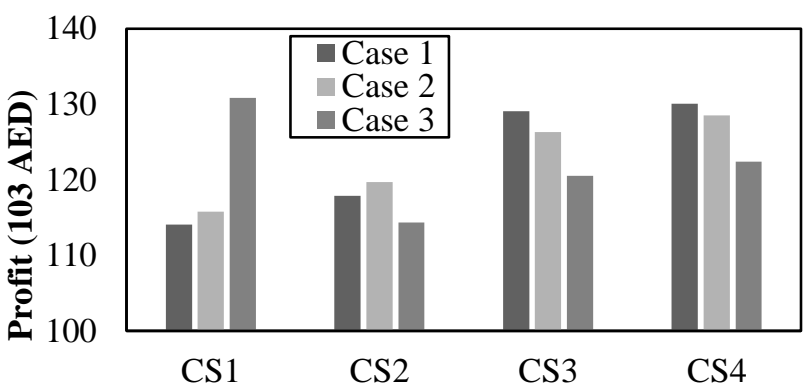

Fig. 5. Profit of CSs in different cases

For the third case study we have considered the incentive provided by the DSO to reduce the load stress on the distribution network during peak hours.

This increased number of EVs will result in high electricity demand. Technically, this would have a negative impact on the distribution grid. Therefore, the power limit on distribution line is considered. Such a constraint would influence the charging game as demonstrated in Fig. 6. Linking this constraint to the EV density, it can be concluded that the impact of the power line limit would be critical only when the EV density is $4 \mathrm{EV} \mathrm{s} / \mathrm{km}$. Under this condition EVs' movements are towards CS 1 and CS 2 due to the availability of power as the limit is not exceed. Profits of CS 3 and CS 4 would start to decrease as the number of EVs requiring the charge decreases.

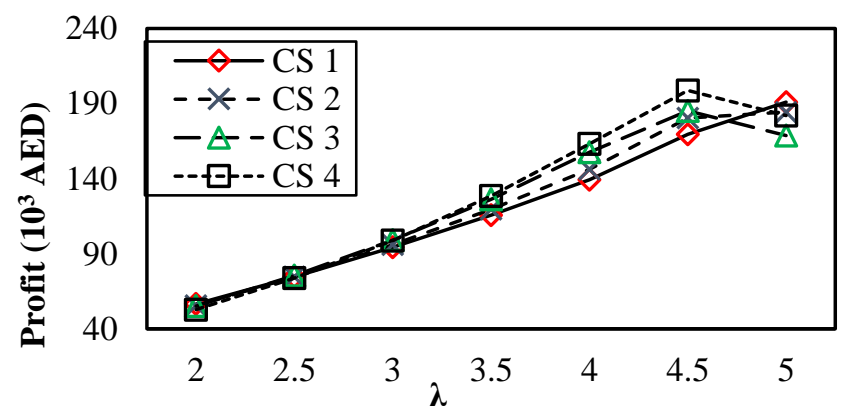

Fig. 6. Profit with respect to EV density with power line limit

\section{CONCLUSION}

In this paper, the price competition between CSs with consideration of various practical parameters and constraints has been studied. A price adjustment game has been modeled through a non-cooperative game theory approach and unique Nash equilibrium has proved. Various cases studies have been simulated to study the impact of the reputation and waiting time on the price competition game. The effect of DSO incentive is also evaluated and its impact on improving the technical performance of system has been observed. Also, the effect of parameter value on the competitive game had been analyzed. In depth analysis has been conducted by accounting for the effect of power limit of distribution system on to replicate the practical problem in the system.

\section{REFERENCES}

[1] Cazzola, P., Gorner, M., Schuitmaker, R. and Maroney, E., "Global EV Outlook 2018," International Energy Agency (IEA): Paris, French, pp. 1-139, 2018.

[2] "Electric Power Monthly" [Online]. Available: https://www.eia.gov/electricity/monthly/current_month/epm.pdf

[3] Sachan S, Amini MH. "Optimal allocation of EV charging spots along with capacitors in smart distribution network for congestion management". Int Trans Electr Energ Syst, Wiley, 2020; 1-14. https://doi.org/10. 1002/2050-7038.12507 
[4] Sachan, Sulabh, Sanchari Deb, and Sri Niwas Singh. "Different charging infrastructures along with smart charging strategies for electric vehicles." Sustainable Cities and Society 60 (2020): 102238.

[5] K. Wang, Z. Ouyang, R. Krishnan, L. Shu and L. He, "A Game TheoryBased Energy Management System Using Price Elasticity for Smart Grids," IEEE Transactions on Industrial Informatics, vol. 11, no. 6, pp. 1607-1616, Dec. 2015.

[6] Y. Liu, R. Deng and H. Liang, "A Stochastic Game Approach for PEV Charging Station Operation in Smart Grid," IEEE Transactions on Industrial Informatics, vol. 14, no. 3, pp. 969-979, March 2018.

[7] Sachan, Sulabh, and Nadia Adnan. "Stochastic charging of electric vehicles in smart power distribution grids." Sustainable cities and society 40 (2018): 91-100.

[8] Sachan, S., et al.: Planning and operation of EV charging stations by chicken swarmoptimization driven heuristics. Energy Convers. Econ. 2,91-99 (2021). https://doi.org/10.1049/enc2.12030.

[9] F. Malandrino, C. Casetti, C.-F. Chiasserini, and M. Reineri, "A game theory analysis of charging stations selection by EV drivers," Performance Evaluation, vol. 83, pp. 16-31, Jan. 2015.

[10] W. Lee, L. Xiang, R. Schober, and V. W. S. Wong, "Electric vehicle charging stations with renewable power generators: A game theoretical analysis," IEEE Transaction on Smart Grid, vol. 6, no. 2, pp. 608-617, Mar. 2015.

[11] O. Beaude, S. Lasaulce, M. Hennebel, and I. Mohand-Kaci, "Reducing the impact of EV charging operations on the distribution network," IEEE Transaction on Smart Grid, vol. 7, no. 6, pp. 2666-2679, Nov. 2016.

[12] Naohiro Uchiyama and Eiichi Taniguchi, "A study of dispatcher's route choice model based on evolutionary game theory," Social and Behavioral Sciences, Volume 39, Pages 495-509, 2012.
[13] H. Yang, S. Yang, Y. Xu, E. Cao, M. Lai and Z. Dong, "Electric Vehicle Route Optimization Considering Time-of-Use Electricity Price by Learnable Partheno-Genetic Algorithm," IEEE Transactions on Smart Grid, vol. 6, no. 2, pp. 657-666, March 2015.

[14] S. Shao, W. Guan and J. Bi, "Electric vehicle-routing problem with charging demands and energy consumption," IET Intelligent Transport Systems, vol. 12, no. 3, pp. 202-212, 42018.

[15] H. Yang, Y. Deng, J. Qiu, M. Li, M. Lai and Z. Y. Dong, "Electric Vehicle Route Selection and Charging Navigation Strategy Based on Crowd Sensing," IEEE Transactions on Industrial Informatics, vol. 13, no. 5, pp. 2214-2226, Oct. 2017.

[16] T. Chen, B. Zhang, H. Pourbabak, A. Kavousi-Fard and W. Su, "Optimal Routing and Charging of an Electric Vehicle Fleet for High-Efficiency Dynamic Transit Systems," IEEE Transactions on Smart Grid, vol. 9, no. 4, pp. 3563-3572, July 2018.

[17] H. Miao, G. Chen, C. Li, Z. Y. Dong and K. P. Wong, "Operating Expense Optimization for EVs in Multiple Depots and Charge Stations Environment Using Evolutionary Heuristic Method," IEEE Transactions on Smart Grid, vol. 9, no. 6, pp. 6599-6611, Nov. 2018.

[18] W. Wei, L. Wu, J. Wang and S. Mei, "Network Equilibrium of Coupled Transportation and Power Distribution Systems," IEEE Transactions on Smart Grid, vol. 9, no. 6, pp. 6764-6779, Nov. 2018.

[19] J. Tan and L. Wang, "Real-time charging navigation of electric vehicles to fast charging stations: A hierarchical game approach," IEEE Transaction on Smart Grid, vol. 8, no. 2, pp. 846-856, Mar. 2017.

W. Yuan, J. Huang, and Y. J. Zhang, "Competitive charging station pricing for plug-in electric vehicles," IEEE Transaction on Smart Grid, vol. 8, no. 2, pp. 627-639, Mar. 2017. 